\title{
AUTOMORPHISMS OF FUNCTIONS IN ABELIAN PERMUTATION GROUPS
}

\author{
by STEPHEN D. COHEN and GARY L. MULLEN
}

(Received 25 November, 1974)

1. Let $\Omega=H_{1} \oplus \ldots \oplus H_{n}$ be an abelian group of permutations of a finite non-empty set $S$. If $H_{i}$ is generated by $\phi_{i}$, let $s_{\phi_{i}}(\alpha)$ denote the length of the cycle of $\phi_{i}$ containing $\alpha$. For any function $f$ on $S$, let $A(f, \Omega)=\{\phi \in \Omega \mid f \phi=f)$. In Theorem 2 we show that, if for every $i \neq j$ and $\alpha \in S, s_{\phi_{1}}(\alpha)$ and $s_{\phi_{j}}(\alpha)$ are relatively prime, then $A(f, \Omega)=A\left(f, H_{1}\right) \oplus \ldots \oplus A\left(f, H_{n}\right)$ for all $f$, while in Theorem 3 we prove the natural converse.

2. Let $\Omega$ be a group of permutations of a finite non-empty set $S$. Let $\Gamma$ be the set of all functions from $S$ into $T$ where $T$ is a finite set containing at least two elements. If $f, g \in \Gamma$, then $f$ is equivalent to $g$ relative to $\Omega$ if there exists a $\phi \in \Omega$ such that $f \phi=g$. We say that a permutation $\phi \in \Omega$ is an automorphism of a function $f$ relative to $\Omega$ if $f \phi=f$. Let $A(f, \Omega)$ denote the group of automorphisms of the function $f$ relative to $\Omega$. For example, if $K$ is the finite field of order $q, S=K^{r}$ where $r \geqq 1, T=K$ and $\Gamma=K\left[x_{1}, \ldots, x_{r}\right]$, then the above situation reduces to that considered by Carlitz in [1].

If $T=\left\{\alpha_{1}, \ldots, \alpha_{v}\right\}$ and $f \in \Gamma$, let $S_{i}=\left\{\beta \in S \mid f(\beta)=\alpha_{i}\right\}$. We define $\pi_{f}$, the partition of $f$, to be the collection of non-empty $S_{i}$ 's. If $f, g \in \Gamma$ with $\pi_{f}=\left\{S_{i}\right\}$ and $\pi_{g}=\left\{T_{i}\right\}$, then $f$ is equivalent to $g$ relative to $\Omega$ if and only if there exists a $\phi \in \Omega$ such that $\phi\left(S_{i}\right) \subseteq T_{i}$ for $i=1, \ldots, v$. If we let $g=f$ we may easily prove

LEMMA 1. If $\phi$ is a permutation of $S$, then $\phi$ is an automorphism of a function $f$ if and only if the cycles of $\phi$ (regarded as sets) form a refinement of $\pi_{f}$.

Suppose now that $\Omega$ is abelian and that $\Omega=H_{1} \oplus \ldots \oplus H_{n}$ where each $H_{i}$ is cyclic generated by $\phi_{i}$. If $\phi \in \Omega$ and $\alpha \in S$, let $\sigma_{\phi}(\alpha)$ denote the cycle of $\phi$ containing $\alpha$ and $s_{\phi}(\alpha)$ the length of $\sigma_{\phi}(\alpha)$.

THEOREM 2. Let $\Omega$ be as above. If for every $i \neq j$ and $\alpha \in S, s_{\phi_{i}}(\alpha)$ and $s_{\phi_{j}}(\alpha)$ are relatively prime, then

for all $f \in \Gamma$.

$$
A(f, \Omega)=A\left(f, H_{1}\right) \oplus \ldots \oplus A\left(f, H_{n}\right)
$$

Proof. Clearly $A\left(f, H_{1}\right) \oplus \ldots \oplus A\left(f, H_{n}\right) \subseteq A(f, \Omega)$ and, if $\psi_{i} \in H_{i}, \psi_{j} \in H_{j}$, then $s_{\psi_{i}}(\alpha)$ and $s_{\psi_{j}}(\alpha)$ are relatively prime. Let $\alpha \in S$ and $\psi \in A(f, \Omega)$ so that $f \psi=f \psi_{1} \ldots \psi_{n}=f$ and hence $f\left(\psi_{1}^{l} \ldots \psi_{n}^{l}(\alpha)\right)=f(\alpha)$ for any integer $l$. By hypothesis and the Chinese Remainder Theorem, we may choose for each $i$ an integer $l_{i}$ such that $l_{i} \equiv 1\left(\bmod s_{\psi_{i}}(\alpha)\right)$ and $l_{i} \equiv 0\left(\bmod s_{\psi}(\alpha)\right)$ for $j \neq i$. Hence $\psi_{1}^{l_{i}} \ldots \psi_{n}^{l_{i}}(\alpha)=\psi_{i}(\alpha)$ so that $f\left(\psi_{i}(\alpha)\right)=f(\alpha)$, which implies that $\psi_{i} \in A\left(f, H_{i}\right)$.

THEOREM 3. If $\Omega$ is as above and (1) holds for all $f \in \Gamma$, then for every $i \neq j$ and $\alpha \in S, s_{\phi_{i}}(\alpha)$ and $s_{\phi_{j}}(\alpha)$ are relatively prime. 
Proof. Suppose that for some $i \neq j$ and some $\alpha \in S,\left(s_{\phi_{i}}(\alpha), s_{\phi_{j}}(\alpha)\right)=s>1$. Let $\psi_{i}=\phi_{i}^{s \phi_{i}(\alpha) / s}$ and $\psi_{j}=\phi_{j}^{s_{\phi_{j}}(\alpha) / s}$ so that $\psi_{i} \in H_{i}, \psi_{j} \in H_{j}$ and $s_{\psi_{i}}(\alpha)=s_{\psi_{j}}(\alpha)=s$.

Case 1. $\sigma_{\psi i}(\alpha)=\sigma_{\psi j}(\alpha)$ (as sets). Then there exists an integer $k$ such that $\psi_{i} \psi_{j}^{-k}(\alpha)=\alpha$. Let $\psi=\psi_{i} \psi_{j}^{-k}$ so that $\sigma_{\psi}(\alpha)=(\alpha)$. Let $S_{1}=\{\alpha\}, S_{2}=S \backslash S_{1}, \pi=\left\{S_{1}, S_{2}\right\}$ and $f$ be any function whose partition is $\pi$. Then by Lemma $1, f \psi=f \psi_{i} \psi_{j}^{-k}=f$ so that $\psi_{i} \psi_{j}^{-k} \in A(f, \Omega)$. Since $\sigma_{\psi_{i}}(\alpha) \notin S_{1}$, then $\psi_{i} \notin A\left(f, H_{i}\right)$ so that (1) fails to hold.

Case 2. $\sigma_{\psi i}(\alpha) \neq \sigma_{\psi}(\alpha)$. Let $\psi=\psi_{i} \psi_{j}$ so that $\left(\psi_{i} \psi_{j}\right)^{s}(\alpha)=\alpha$ which implies that $s_{\psi}(\alpha) \leqq s$. Hence $\sigma_{\psi}(\alpha)$ and $\sigma_{\psi}(\alpha)$ cannot both be contained in $\sigma_{\psi}(\alpha)$, so that we may assume that $\sigma_{\psi_{i}}(\alpha) \nsubseteq \sigma_{\psi}(\alpha)$. Let $S_{1}=\sigma_{\psi}(\alpha), S_{2}=S \backslash S_{1}, \pi=\left\{S_{1}, S_{2}\right\}$ and $f$ be any function whose partition is $\pi$. Then $\psi=\psi_{i} \psi_{j} \in A(f, \Omega)$; but $\psi_{i} \notin A\left(f, H_{i}\right)$, so that again (1) fails to hold.

\section{REFERENCE}

1. L. Carlitz, Invariantive theory of equations in a finite field, Trans. Amer. Math. Soc. 75 (1953), 405-427.

UNIVERSITY OF GLASGOW

GlasGow G12 8QW
The Pennsylvania State University

Shenango Valley Campus

147 SHENANGo AVENUE

SHARON

Pennsylvania 16146, U.S.A. 